Collectanea Mathematica (electronic version): http://www.imub.ub.es/collect

Collect. Math. 44 (1993), 147-154

(C) 1994 Universitat de Barcelona

\title{
Higher order Hardy inequalities
}

\author{
Alois Kufner
}

Math. Institute, Czech. Acad. Sci.,

Zitna 25, 11567 Praha 1, Czech Republic

\section{AbSTRACT}

This note deals with the inequality

$$
\left(\int_{a}^{b}|u(x)|^{q} w_{0}(x) d x\right)^{1 / q} \leq C\left(\int_{a}^{b}\left|u^{(k)}(x)\right|^{p} w_{k}(x) d x\right)^{1 / p},
$$

more precisely, with conditions on the parameters $p>1, q>0$ and on the weight functions $w_{0}, w_{k}$ (measurable and positive almost everywhere) which ensure that (1) holds for all functions $u$ from a certain class $K$ with a constant $C>0$ independent of $u$.

Here $-\infty \leq a<b \leq \infty$ and $k \in \mathbb{N}$ and we will consider classes $K$ of functions $u=u(x)$ defined on $(a, b)$ whose derivatives of order $k-1$ are absolutely continuous and which satisfy the "boundary conditions"

$$
\begin{array}{lll}
u^{(i)}(a)=0 & \text { for } & i \in M_{0}, \\
u^{(j)}(b)=0 & \text { for } & j \in M_{1}
\end{array}
$$

where $M_{0}, M_{1}$ are subsets of the set $M=\{0,1, \ldots, k-1\}$; we will suppose that the number of conditions in (2) is exactly $k$. This class will be denoted by

$$
A C^{(k-1)}\left(a, b ; M_{0}, M_{1}\right) \text {. }
$$

The conditions (2) are reasonable since they allow to exclude functions like polynomials of order $\leq k-1$ for which the right hand side in (1) is zero while the left hand side is positive. 
Let us start with some remarks.

(i) We will concentrate on the case

$$
k>1
$$

since for $k=1$ the problem is completely solved: see, e.g., the book Opic, Kufner [4], Chapter 1. Some particular results concerning the case $k=2, k=3$ and - for a special choice of the sets $M_{0}, M_{1}$ - also higher values of $k$ can be found in the paper Kufner, Wannebo [3].

(ii) For $(a, b)=(0, \infty), k \in \mathbb{N}$ arbitrary and $M_{0}=M, M_{1}=\emptyset$ or $M_{0}=$ $\{0,1, \ldots, m-1\}, M_{1}=M \backslash M_{0}, 0<m<k$, the problem is also solved: see Stepanov [5] or Kufner, Heinig [2], respectively. These results cover all reasonable cases when the interval $(a, b)$ is infinite. Therefore, we will concentrate on the case of a finite interval $(a, b)$. Without loss of generality it can be assumed that

$$
(a, b)=(0,1) .
$$

In the sequel, we will make substantial use of some functions and constants. For $r \neq 1$, we will denote

$$
r^{\prime}=\frac{r}{r-1}, \text { i.e. } \frac{1}{r}+\frac{1}{r^{\prime}}=1 .
$$

Further, let us denote for $i=1,2$

$$
\begin{aligned}
& W_{0 i}(t)=w_{0}(t) t^{\alpha_{i} q}(1-t)^{\beta_{i} q}, \\
& W_{k i}(t)=w_{k}^{1-p^{\prime}}(t) t^{\gamma_{i} p^{\prime}}(1-t)^{\delta_{i} p^{\prime}},
\end{aligned}
$$

where $w_{0}(t), w_{k}(t)$ are the weight functions appearing in (1) and $\alpha_{i}, \beta_{i}, \gamma_{i}, \delta_{i}$ $(i=1,2)$ are certain nonnegative integers, and let us introduce functions

$$
\begin{aligned}
& B_{1}(x)=\left(\int_{x}^{1} W_{01}(t) d t\right)^{1 / q}\left(\int_{0}^{x} W_{k 1}(t) d t\right)^{1 / p^{\prime}}, \\
& B_{2}(x)=\left(\int_{0}^{x} W_{02}(t) d t\right)^{1 / q}\left(\int_{x}^{1} W_{k 2}(t) d t\right)^{1 / p^{\prime}}
\end{aligned}
$$

and constants

$$
\begin{aligned}
& A_{1}=\left[\int_{0}^{1}\left(\int_{x}^{1} W_{01}(t) d t\right)^{r / q}\left(\int_{0}^{x} W_{k 1}(t) d t\right)^{r / q^{\prime}} W_{k 1}(x) d x\right]^{1 / r}, \\
& A_{2}=\left[\int_{0}^{1}\left(\int_{0}^{x} W_{02}(t) d t\right)^{r / q}\left(\int_{x}^{1} W_{k 2}(t) d t\right)^{r / q^{\prime}} W_{k 2}(x) d x\right]^{1 / r},
\end{aligned}
$$


where

$$
\frac{1}{r}=\frac{1}{q}-\frac{1}{p}
$$

We suppose that all expressions appearing in formulas (7) - (10) are well defined. Of course, it also depends on the values $\alpha_{i}, \beta_{i}, \gamma_{i}, \delta_{i}$ which have not yet been determined. Later, we will show how these integers can be determined by the sets $M_{0}$ and $M_{1}$ which appear in the conditions (2).

If we suppose for a moment that these integers are known, then the main result can be formulated as follows:

\section{Proposition 1}

Let $M_{0}, M_{1}$ be two nonempty subsets of the set $\{0,1, \ldots, k-1\}$ containing together $k$ elements. Let $\alpha_{i}, \beta_{i}, \gamma_{i}, \delta_{i}, \quad i=1,2$, be nonnegative integers corresponding to the pair $M_{0}, M_{1}$. Let $w_{0}(t), w_{k}(t)$ be weight functions defined on $(0,1)$ and let

$$
1<p<\infty, 0<q<\infty, q \neq 1
$$

Then the (HARDY) inequality

$$
\left(\int_{0}^{1}|u(t)|^{q} w_{0}(t) d t\right)^{1 / q} \leq C\left(\int_{0}^{1}\left|u^{(k)}(t)\right|^{p} w_{k}(t) d t\right)^{1 / p}
$$

holds for every function $u \in A C^{(k-1)}(0,1)$ satisfying the conditions

$$
\begin{array}{ll}
u^{(i)}(0)=0 & \text { for } \quad i \in M_{0}, \\
u^{(j)}(1)=0 & \text { for } j \in M_{1}
\end{array}
$$

if and only if

$$
\sup _{0<x<1} B_{i}(x)=B_{i}<\infty, \quad i=1,2
$$

in the case $p \leq q$, and

$$
A_{i}<\infty, \quad i=1,2
$$

in the case $p>q$, where $B_{i}(x)$ and $A_{i}$ are given by formulas (7) - (11). 
Determination of the integers $\alpha_{1}, \ldots, \delta_{2}$.

Let us consider a very simple boundary value problem

$$
\begin{array}{rll}
u^{(k)}=f & \text { in } \quad(0,1), \\
u^{(i)}(0)=0 & \text { for } \quad i \in M_{0}, \\
u^{(j)}(1)=0 & \text { for } \quad j \in M_{1}
\end{array}
$$

where $f$ does not change the sign in $(0,1)$ and $M_{0}, M_{1}$ are the subsets of $M=$ $\{0,1, \ldots, k-1\}$ mentioned in Proposition 1 .

Suppose that the solution $u$ can be expressed uniquely in the form

$$
u(x)=\int_{0}^{x} K_{1}(x, t) f(t) d t+\int_{x}^{1} K_{2}(x, t) f(t) d t .
$$

The kernels $K_{1}(x, t), K_{2}(x, t)$ are then polynomials. We will write

$$
K_{i}(x, t) \approx x^{\alpha_{i}}(1-x)^{\beta_{i}} t^{\gamma_{i}}(1-t)^{\delta_{i}}
$$

if there exist positive constants $c_{1}, c_{2}$ such that the estimates

$$
c_{1} \leq \frac{K_{i}(x, t)}{x^{\alpha_{i}}(1-x)^{\beta_{i}} t^{\gamma_{i}}(1-t)^{\delta_{i}}} \leq c_{2}
$$

hold for $0<t<x<1 \quad(i=1)$ and $0<x<t<1 \quad(i=2)$, respectively.

Now, we will show under what conditions (18) is fulfilled. For this purpose, let us split the set $M=\{0,1, \ldots, k-1\}$ into $s$ successive groups $G_{1}, G_{2}, \ldots, G_{s}(s \geq 2)$ according to the following scheme:

$$
\begin{array}{ccl}
G_{1}=\{0,1, \ldots, m-1\} & \left(k_{1}\right. \text { elements, } & \left.k_{1}=m\right), \\
G_{2}=\{m, m+1, \ldots, n-1\} & \left(k_{2}\right. \text { elements, } & \left.k_{2}=n-m\right), \\
G_{3}=\{n, n+1, \ldots, r-1\} & \left(k_{3}\right. \text { elements, } & \left.k_{3}=r-n\right), \\
& \cdot & \\
G_{s}=\{h, h+1, \ldots, k-1\} & \left(k_{s}\right. \text { elements, } & \left.k_{s}=k-h\right),
\end{array}
$$

(i.e. $G_{i}$ has $k_{i}$ elements, $k_{i}>0, i=1,2, \ldots, s$, and $k_{1}+k_{2}+\ldots+k_{s}=k$ ), and suppose that the sets $M_{0}$ and $M_{1}$ appearing in the boundary conditions in (16) are defined as follows:

$$
\begin{gathered}
M_{0}=G_{1} \cup G_{2} \cup \ldots \cup G_{s-1}, \quad M_{1}=G_{2} \cup G_{4} \cup \ldots \cup G_{s} \text { for } s \text { even. } \\
M_{0}=G_{1} \cup G_{2} \cup \ldots \cup G_{s}, \quad M_{1}=G_{2} \cup G_{4} \cup \ldots \cup G_{s-1} \text { for } s \text { odd. }
\end{gathered}
$$

Then we have 


\section{Proposition 2}

If the set $M=\{0,1, \ldots, k-1\}$ is splitted into $s$ groups according to (19), the sets $M_{0}$ and $M_{1}$ are defined by (20) and (21) and the solution $u$ to the boundary value problem (16) can be expressed in the form (17), then

$$
\begin{aligned}
& K_{1}(x, t) \approx x^{k_{1}-1} t^{k_{2}}, \quad K_{2}(x, t) \approx x^{k_{1}} t^{k_{2}-1} \text { for } s=2, \\
& K_{i}(x, t) \approx x^{k_{1}}(1-t)^{k_{s}}, \quad i=1,2, \text { for } s \text { odd } \\
& K_{i}(x, t) \approx x^{k_{1}} t^{k_{s}}, \quad i=1,2, \text { for } s>2 \text { even } .
\end{aligned}
$$

Remarks. (i) The proof of Proposition 2 is elementary but cumbersome. It is based on the fact that the solution $u$ to the boundary value problem (16) can be expressed in the form

$$
\begin{aligned}
& u(x)=c_{o} \int_{0}^{x}\left(x-t_{1}\right)^{k_{1}-1} \int_{t_{1}}^{1}\left(t_{2}-t_{1}\right)^{k_{2}-1} \int_{0}^{t_{2}}\left(t_{2}-t_{3}\right)^{k_{3}-1} \ldots \\
& \ldots F\left(t_{s-1}\right) d t_{s-1} \ldots d t_{2} d t_{1}
\end{aligned}
$$

where $c_{0}=\left[\left(k_{1}-1\right) !\left(k_{2}-1\right) ! \ldots\left(k_{s}-1\right) !\right]^{-1}$ and $F\left(t_{s-1}\right)$ is either

$$
\int_{t_{s-1}}^{1}\left(t_{s}-t_{s-1}\right)^{k_{s}-1} f\left(t_{s}\right) d t_{s} \quad \text { for } s \text { even }
$$

or

$$
\int_{0}^{t_{s-1}}\left(t_{s-1}-t_{s}\right)^{k_{s}-1} f\left(t_{s}\right) d t_{s} \text { for } s \text { odd } .
$$

For $s=2$, it can be found in the paper [3], for $s>2$ in the preprint [1].

(ii) In (20), (21) we have always assumed that the first group $G_{1}$ belongs to $M_{0}$ so that we start with the boundary condition $u(0)=0,0 \in M_{0}$. If we suppose that $0 \in M_{1}$, i.e. that the boundary condition $u(1)=0$ appears in (16), and have

$$
M_{0}=G_{2} \cup G_{4} \cup \ldots, \quad M_{1}=G_{1} \cup G_{3} \cup \ldots,
$$

then we simply exchange the role of the sets $M_{0}$ and $M_{1}$, i.e. of the endpoints $x=0$ and $x=1$, and a corresponding assertion holds again, if we replace in (22) $x$ by $1-x$ and $t$ by $1-t$.

(iii) In the foregoing cases, we have assumed that

$$
M_{0} \cup M_{1}=M \text {, i.e. } \quad M_{0} \cap M_{1}=\emptyset .
$$


If the sets $M_{0}$ and $M_{1}$ again have together $k$ elements, but have a nonempty intersection, then the method described above cannot be used. Nonetheless, many examples allow to expect that - provided there is a unique representation of the solution $u$ of (16) in the form (17) - the kernels $K_{i}(x, t)$ again behave according to (18). Therefore, let us formulate the following conjecture:

Suppose that $M_{0} \cap M_{1} \neq \emptyset$.

(a) Define $\widetilde{M}_{1}$ by

$$
\widetilde{M}_{1}=M \backslash M_{0} .
$$

Then the pair $M_{0}, \widetilde{M}_{1}$ satisfies the conditions of either Proposition 2 (if $G_{1} \subset M_{0}$ ) or of part (ii) of this Remark (if $G_{1} \subset \widetilde{M}_{1}$ ), and consequently, the kernels $K_{i}^{(a)}(x, t)$ corresponding to the pair $M_{0}, \widetilde{M}_{1}$ satisfy (18): There are positive integers $\alpha_{i}(a), \beta_{i}(a), \gamma_{i}(a), \delta_{i}(a)$ such that

$$
K_{i}^{(a)}(x, t) \approx x^{\alpha_{i}(a)}(1-x)^{\beta_{i}(a)} t^{\gamma_{i}(a)}(1-t)^{\delta_{i}(a)}, \quad i=1,2 .
$$

(b) Define $\widetilde{M}_{0}$ by

$$
\widetilde{M}_{0}=M \backslash M_{1} .
$$

Then the pair $\widetilde{M}_{0}, M_{1}$ again satisfies the conditions which allow to state that for the corresponding kernels $K_{i}^{(b)}(x, t)$ we have

$$
K_{i}^{(b)}(x, t) \approx x^{\alpha_{i}(b)}(1-x)^{\beta_{i}(b)} t^{\gamma_{i}(b)}(1-t)^{\delta_{i}(b)}, \quad i=1,2 .
$$

(c) For the kernels $K_{i}(x, t)$ corresponding to the initial pair $M_{0}, M_{1}$ we have (18) with

$$
\alpha_{i}=\alpha_{i}(a), \beta_{i}=\beta_{i}(b), \gamma_{i}=\gamma_{i}(a), \delta_{i}=\delta_{i}(b) .
$$

Idea of the proof of Proposition 1

We consider the Hardy inequality (12) on the class $A C^{(k-1)}\left(0,1 ; M_{0}, M_{1}\right)$, i.e., for functions $u$ satisfying (13). Therefore, let us consider the boundary value problem (16) and denote by $T$ the operator defined by formula (17):

$$
(T f)(x)=\int_{0}^{x} K_{1}(x, t) f(t) d t+\int_{x}^{1} K_{2}(x, t) f(t) d t .
$$

Since the function $u=T f$ satisfies conditions (13) and $u^{(k)}=f$, we can instead of the inequality (12) investigate the inequality

$$
\left(\int_{0}^{1}|(T f)(x)|^{q} w_{0}(x) d x\right)^{1 / q} \leq C\left(\int_{0}^{1} f^{p}(x) w_{k}(x) d x\right)^{1 / p}
$$

for functions $f \geq 0$. 
Now, it can be shown that the validity of (23) for $f \geq 0$ is equivalent to the validity of the inequalities

$$
\left(\int_{0}^{1}\left|\left(J_{i} f\right)(x)\right|^{q} w_{0}(x) d x\right)^{1 / q} \leq C_{i}\left(\int_{0}^{1} f^{p}(x) w_{k}(x) d x\right)^{1 / p}, \quad i=1,2,
$$

where

$$
\left(J_{1} f\right)(x)=\int_{0}^{x} K_{1}(x, t) f(t) d t, \quad\left(J_{2} f\right)(x)=\int_{x}^{1} K_{2}(x, t) f(t) d t .
$$

But due to (18), the inequalities (24) are equivalent to the inequalities

$$
\begin{gathered}
\left(\int_{0}^{1}\left[x^{\alpha_{1}}(1-x)^{\beta_{1}} \int_{0}^{x} t^{\gamma_{1}}(1-t)^{\delta_{1}} f(t) d t\right]^{q} w_{0}(x) d x\right)^{1 / q} \\
\leq \widetilde{C}_{1}\left(\int_{0}^{1} f^{p}(x) w_{k}(x) d x\right)^{1 / p}
\end{gathered}
$$

and

$$
\begin{gathered}
\left(\int_{0}^{1}\left[x^{\alpha_{2}}(1-x)^{\beta_{2}} \int_{x}^{1} t^{\gamma_{2}}(1-t)^{\delta_{2}} f(t) d t\right]^{q} w_{0}(x) d x\right)^{1 / q} \\
\leq \widetilde{C}_{2}\left(\int_{0}^{1} f^{p}(x) w_{k}(x) d x\right)^{1 / p}
\end{gathered}
$$

respectively, and these last two inequalities can be easily rewritten into the form

$$
\left(\int_{0}^{1}|(H g)(x)|^{q} w(x) d x\right)^{1 / q} \leq \widetilde{C}\left(\int_{0}^{1} g^{p}(x) v(x) d x\right)^{1 / p}
$$

where $H$ is the Hardy operator,

$$
(H g)(x)=\int_{0}^{x} g(t) d t \quad \text { or } \quad(H g)(x)=\int_{x}^{1} g(t) d t .
$$

Finally, necessary and sufficient conditions for the validity of (25) (see, e.g., [4]) lead to the conditions (14) (if $p \leq q$ ) or (15) (if $p>q$ ).

Consequently, the integers $\alpha_{1}, \ldots, \delta_{2}$ which appear in (6) can be determined from the behavior of the kernels $K_{1}(x, t), K_{2}(x, t)$ described by (18). 


\section{References}

1. A. Kufner, Higher order Hardy inequalities, Preprint No. 672, Universität Heidelberg, Sonderforschungsbereich 123, 1992.

2. A. Kufner, H.P. Heinig, Hardy's inequality for higher order derivatives, Trudy Mat. Inst. Steklov 192 (1990), 105-113, (Russian).

3. A. Kufner, A. Wannebo, Some remarks on the Hardy inequality for higher order derivatives. In: General Inequalities VI. International Series of Numerical Mathematics 103. Birkhäuser Verlag, Basel 1992, 33-48.

4. B. Opic, A. Kufner, Hardy-type inequalities, Pitman Research Notes in Mathematics Series 219. Longman Scientific and Technical, Harlow 1990.

5. V.D. Stepanov, Two-weighted estimates for Riemann-Liouville integrals. Preprint No. 39, Math. Inst. Czech. Acad. Sci., Prague 1988. 